Bertelsmann-Studie

\section{Deutschland hat Rücken - mancherorts besonders oft}

— Rückenoperationen haben in den vergangenen Jahren in Deutschland stark zugenommen: Waren es 2007 noch 452.000 Eingriffe, gab es 2015 bereits 772.000 - eine Steigerung um durchschnittlich $71 \%$ Das hat der "Faktencheck Gesundheit" der Bertelsmann-Stiftung ergeben, der die Häufigkeit dreier Rückenoperationen (Bandscheiben-Eingriffe, Dekompressions-OP und Spondylodesen) analysierte. Die Zahlen fallen in den einzelnen Bundesländern jedoch sehr unterschiedlich aus, wie am Beispiel der Versteifungs- und VerblockungsEingriffe zu sehen ist (Abb. 1). Für Versteifungsoperationen sind Thüringen, Hessen und das Saarland OP-Hochburgen, während dieser Eingriff in Sachsen am seltensten vorgenommen wird. Warum die Versorgung regional so unterschiedlich ist, lasse sich mit den vorhandenen Daten zwar nur schwer erklären, räumt die Stiftung ein. Allerdings sei dies ein Indiz für eine unterschiedliche Organisation der Versorgung und Vorgehensweise bei Diagnostik und Therapie. Hierzu passt ein weiteres auffälliges Ergebnis: Seit 2007 hat die Zahl der Patienten, die aufgrund von Rückenschmerzen (ICD-M 54) in die Klinik aufgenommen wurden, von
116.000 auf 200.000 in 2015 zugenommen - auch hier zeigen sich deutliche wohnortabhängige Unterschiede. So gibt es besonders viele Klinikaufenthalte aufgrund der Diagnose in Sachsen-Anhalt (400 je 100.000 Einwohner) - in Hamburg sind es dagegen fast zwei Drittel weniger (135 je 100.000 Einwohner). Laut Bertelsmann-Stiftung lassen sich diese Unterschiede medizinisch nicht erklären. Für Dr. Oliver Emrich, Vizepräsident der Deutschen Gesellschaft für Schmerzmedizin, ist das Ergebnis verständlich: „Dort, wo es noch viele niedergelassene Schmerztherapeuten gibt, ist die Zahl der Klinikaufenthalte wegen Rückenbeschwerden niedrig. Wo diese Ärzte fehlen, gehen die Patienten notgedrungen in die Krankenhäuser. Und die machen das, was sie am besten können: operieren. In 90\% der Fälle ohne ausreichende Indikation."

Auch die Bertelsmann-Stiftung merkt kritisch an, dass die Mehrheit der Patienten im Krankenhaus keine spezifische Schmerztherapie oder Operation erhalte. Es würden lediglich diagnostische Untersuchungen (wie z.B. MRT) gemacht. Diese Maßnahmen könnten jedoch auch ambulant erfolgen, so die Stiftung.

(ajo)

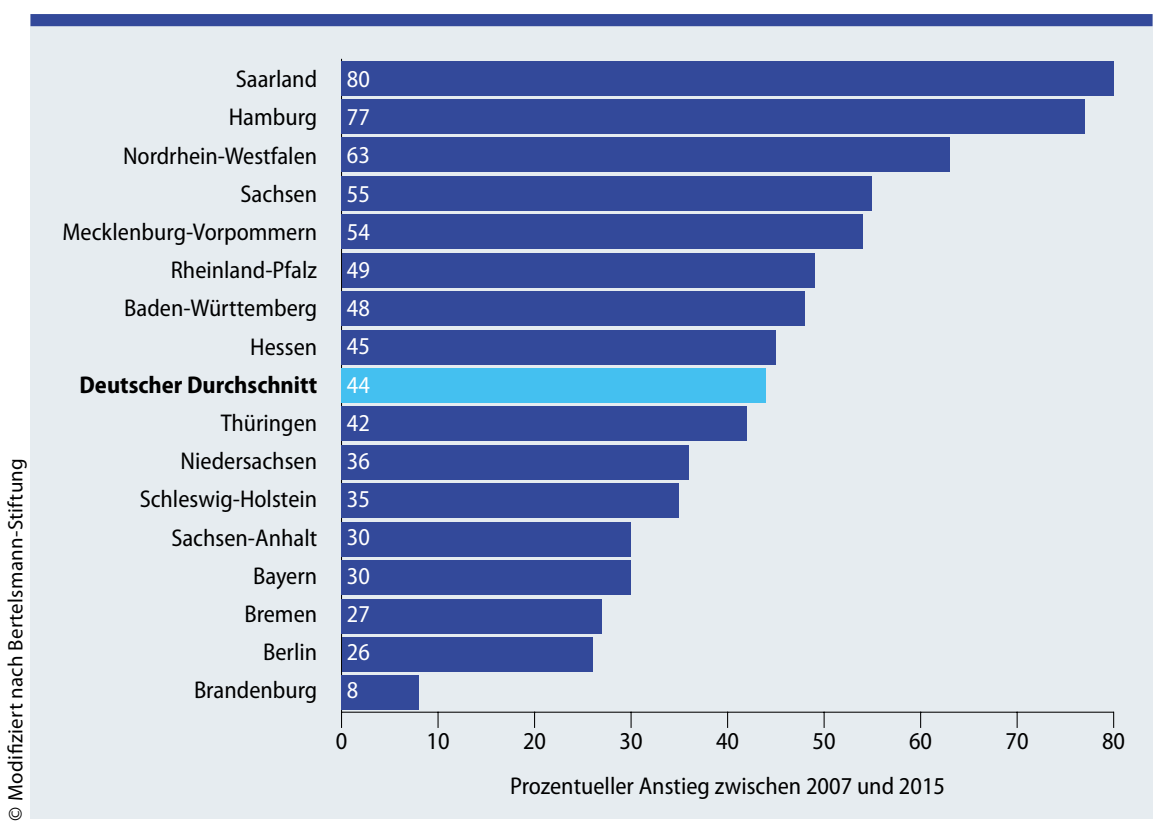

Abb. 1: Anstieg der Verblockungs-Eingriffe und Versteifungen nach Bundesländern.
Verordnungen

\section{Noch keine Zahlen zu Cannabis}

— Dem Bundesgesundheitsministerium (BMG) liegen aktuell noch keine Zahlen zur Verordnung von Cannabis auf Rezept vor. Das geht aus der Antwort des Pressereferenten des BMG, Oliver Ewald, in der Bundespressekonferenz hervor, er könne deshalb auch keine Angaben dazu machen, wie rege Cannabis verordnet wird. Ewald war zuvor auf Medienberichte zu Problemen in der Verordnung von Cannabis angesprochen worden.

Ewald endete allerdings mit einem deutlichen Appell an Ärzte und die Krankenkassen: „Grundsätzlich ist es so, dass die Ärzte Cannabis entsprechend zu verordnen und die Krankenkassen es zu genehmigen haben!"

(mmr)

Werbung für Brausetabletten Vitamin C bringt keine Zusatzindikation

— Ein Arzneihersteller darf nicht mit einer Indikation werben, für die sein Mittel nicht zugelassen ist, so Oberlandesgericht (OLG) Stuttgart. Die Beigabe von Vitamin C zu einem Arzneimittel ermöglicht dem Hersteller nicht automatisch die Werbebotschaft „unterstützt das Immunsystem“. Das OLG untersagte damit die Werbung eines Generika-Herstellers für seine Brausetabletten "ASS + C".

Die als Schmerzmittel zugelassenen Brausetabletten enthalten 600 Milligramm Acetylsalicylsäure und 200 Milligramm Ascorbinsäure. Der Hersteller bewarb die Tabletten mit der Aussage: „Wirkt mit Acetylsalicylsäure als Brausetablette rasch gegen Schmerzen. Eine Extraportion Vitamin C unterstützt das Immunsystem." Dagegen klagte ein Wettbewerbsverband. Der Verweis auf den Schutz des Immunsystems sei hier unzulässig, urteilten die Stuttgarter Richter.

(mwo) 